Gabi Schneider*

\title{
Wissenschaftliche Information: Zugang, Verarbeitung und Speicherung
}

\author{
Ein Förderprogramm zur Bündelung der wissenschaftlichen Information in der Schweiz
}

DOI 10.1515/bfp-2016-0069

Zusammenfassung: Das Förderprogramm „Wissenschaftliche Information: Zugang, Verarbeitung und Speicherung“ (SUK P-2) baut für die Schweizer Wissenschaft ein Netzwerk von Diensten auf, das einen einfachen Zugang zu Publikationen und Daten ermöglicht und Werkzeuge für deren Bearbeitung und Speicherung anbietet. Die Lösungen verbinden die Leistungen von Bibliotheken, ITServices und Scientific IT zu einer Basis für Science 2.0 und Open Science. Für den dauerhaften Betrieb wird eine hochschulübergreifende Governance aufgebaut.

Schlüsselwörter: Schweiz; swissuniversities; Forschungsinfrastrukturen; wissenschaftliche Information; Digitalisierung

\section{Scientific Information: Access, Processing and Safeguarding. A Program Combining Efforts to Manage Scientific Information in Switzerland}

Abstract: The program "Scientific information: Access, processing and safeguarding” (SUC P-2) is building a network of services for Swiss science that will enable easy access to publications and data and provide tools for their processing and storage. These solutions combine the services of libraries, IT services and scientific IT to form a foundation for Science 2.0 and Open Science. Central governance is being created to maintain continuous operation.

Keywords: Switzerland; swissuniversities; research infrastructures; scholarly information; digitization

Inhalt

1 Förderung von Forschungsinfrastrukturen in der Schweiz .................... 424

2 DasProgramm,,Wissenschaftliche Information: Zugang, Verarbeitung undSpeicherung“"(SUK P-2) 425

*Kontaktperson: Gabi Schneider,

gabi.schneider@swissuniversities.ch
3 Ausgangslage, Vision und Mission ... . . . . 426

4 Projekteingaben und Mandate . . . . . . . . 426

5 StrategischeHauptstoßrichtungen und Projekte . 427

5.1 „Publikationen“: Lizenzierung, Open Access, Digitalisierung, Metadata/Search . . . . . . . 427

5.2 „E-Science“: Data Lifecycle Management, Training und Support . . . . . . . . . . . . . . . . 429

5.3 „Basis“: Kernstücke des künftigen Service-Networks .................... . 430

5.4 „Dienste“: konsolidieren und erweitern . . . . . . 431

6 Ausbau der Projektcluster (2017 bis 2020) . . . . 432

7 Von der Programmorganisation zu einer „nationalen Organisation“ . . . . . . . . . . . . . 432

8 Programmfortsetzung 2017 bis 2020 . . . . . . . 432

\section{Förderung von Forschungsinfrastrukturen in der Schweiz}

Gemäß Bundesverfassung Artikel 63a ${ }^{1}$ sorgen in der Schweiz Bund und Kantone gemeinsam für einen wettbewerbsfähigen, qualitativ hochstehenden Hochschulraum. Dieser besteht aus Universitären Hochschulen (den Eidgenössischen Technischen Hochschulen und den kantonalen Universitäten), Fachhochschulen, Pädagogischen Hochschulen und anderen Institutionen des Hochschulbereichs. Die Zusammenarbeit wird durch das neue Hochschulförderungs- und Koordinationsgesetz (HFKG) geregelt. Während die Bestimmungen die Organe und die Akkreditierung betreffend seit Anfang 2015 in Kraft sind, gelten die Finanzierungsbestimmungen ab 2017. ${ }^{2}$

2011 erarbeitete das Staatssekretariat für Bildung, Forschung und Innovation (SBFI) als Reaktion auf die euro-

1 Bundesverfassung der Schweizerischen Eidgenossenschaft (SR 101). 2 Vgl. die Informationen auf den Webseiten des Staatssekretariats für Bildung, Forschung und Innovation (SBFI): https://www.sbfi.ad min.ch/sbfi/de/home/themen/hochschulen/hfkg.html. 
päische ESFRI-Roadmap ${ }^{3}$ von 2008 die erste Schweizer Roadmap für Forschungsinfrastrukturen (FIS), um die langfristige Finanzierung nationaler und internationaler Vorhaben besser zu planen. Mit der zweiten Roadmap $2015,{ }^{4}$ die als Grundlage für die Legislaturperiode 2017 bis 2020 dient und stärker auf FIS mit einem Schweizer Fokus ausgerichtet ist, soll eine kohärente Abstimmung zwischen nationaler und internationaler Forschungsförderung und mit den Prioritäten der Hochschulen erreicht werden. ${ }^{5}$

Unter Forschungsinfrastrukturen werden Einrichtungen verstanden, die einen wissenschaftlichen Mehrwert liefern, die von mehreren Institutionen genutzt werden und die grundsätzlich für nationale und internationale Teilnehmer offen stehen. Organisatorisch können sie an einen Standort gebunden oder als Netzwerk mit zentraler Managementstruktur aufgebaut sein. Sie sind langfristig angelegt ( $>10$ Jahre) und verfolgen in der Regel keine selbständige Forschungstätigkeit. Die aktuelle Roadmap 2015 zählt in Anhang B rund 70 bestehende FIS auf. In diesem Inventar wird das Programm „Wissenschaftliche Information: Zugang, Verarbeitung und Speicherung“ (SUK P-2) ${ }^{6}$ unter dem Typ „technische Infrastrukturen - e-Infrastrukturen" geführt.

Das Programm wird aus sogenannten Projektgebundenen Beiträgen alimentiert. Mit diesem Förderinstrument unterstützt der Bund universitäre Kooperations- und Innovationsprojekte von gesamtschweizerischer Bedeutung und Programme zu spezifischen Themen. ${ }^{7}$ Die Förderung basiert auf dem Prinzip der Anschubfinanzierung: Projekteingaben, die dem definierten Rahmen eines Programms entsprechen, erhalten eine Finanzierung von $50 \%$ der Projektsumme. Aus den geförderten Vorhaben sollen selbständige Infrastrukturen entstehen. Auf dieser Basis wurde im Jahr 2000 zum Beispiel das Konsortium der Schweizer Hochschulbibliotheken initiiert, das Konsortiallizenzen

\footnotetext{
3 European Strategy Forum for Research Infrastructures ESFRI. Für die aktuelle Roadmap 2016 vgl. http://www.esfri.eu/roadmap-2016. 4 Schweizer Roadmap für Forschungsinfrastrukturen im Hinblick auf die BFI-Botschaft 2017-2020: https://www.sbfi.admin.ch/sbfi/de/ho me/themen/forschung-und-innovation-in-der-schweiz/schweizer-roa dmap-fuer-forschungsinfrastrukturen.html.

5 Vgl. Art. 41 des Bundesgesetzes über die Förderung der Forschung und der Innovation (FIFG, SR 420.1) und die zugehörige Verordnung (V-FIFG, SR 420.11), Art. 55.

6 Der „Code“ SUK P-2 bezeichnet das Programm als Nr. 2 von insgesamt zehn laufenden thematischen Programmen in der Förderperiode 2013-2016.

7 Zum Förderinstrument der projektgebundenen Beiträge vgl. https:// www.sbfi.admin.ch/sbfi/de/home/themen/hochschulen/universitae ten/bundesbeitraege-fuer-zusammenarbeitsprojekte-der-kantonalenuniv.html.
}

für die Schweizer Hochschulbibliotheken verhandelt und seit 2006 selbsttragend ist. ${ }^{8}$

\section{Das Programm, Wissenschaftliche Information: Zugang, Verarbeitung und Speicherung“(SUK P-2)}

Das Programm Wissenschaftliche Information baut auf den Errungenschaften von zwei Vorgängerprogrammen auf: E-lib.ch, das in Bibliothekskreisen einen hohen Bekanntheitsgrad erreicht hat, und SWITCH/AAA. Während E-lib.ch unter der Leitung der ETH-Bibliothek erfolgreich digitale Infrastrukturen im bibliotheksnahen Bereich lancierte, ${ }^{9}$ baute der Netzwerkanbieter der Schweizer Hochschulen, SWITCH, ${ }^{10}$ mit dem Programm SWITCH/AAA nationale IT-Infrastrukturen in den Bereichen E-Learning, Grid-Computing und virtuelle Organisationen auf. ${ }^{11}$ Das Programm SUK P-2 soll in einem nächsten Schritt kooperative Dienste im Bereich der wissenschaftlichen Information als übergreifendes Aufgabenfeld der Bibliotheken und der IT-Dienstleister (IT-Services, Scientific IT, SWITCH) der Hochschulen zusammenführen. Die Programmleitung ist bei swissuniversities, seit Inkrafttreten des HFKG die Rektorenkonferenz aller Hochschultypen, angesiedelt. ${ }^{12}$

Als Ausführungsgrundlage wurden im Jahr 2013 in einem breit angelegten Bottom-up-Prozess zunächst die Strategiedokumente und das Projektauswahlverfahren erarbeitet. Das Dokument „Bündelung der Kräfte in der wissenschaftlichen Information“ hält die nationale Strategie des Programms fest, während das „White Paper for a Swiss Information Provisioning and Processing Infrastructure 2020" die Umsetzungsstrategie darlegt. ${ }^{13}$ Die nationale Strategie wurde von der damaligen Schweizerischen Universitätskonferenz (SUK) am 3. April 2014 bewilligt. Am 11. April 2014 entschied der Lenkungsausschuss des Programms über die ersten Projekteingaben.

8 Konsortium der Schweizer Hochschulbibliotheken unter http:// www.consortium.ch.

9 http://www.e-lib.ch.

10 SWITCH unter https://www.switch.ch.

11 https://projects.switch.ch/aaa.

12 Swissuniversities unter http://www.swissuniversities.ch.

13 Die genannten Dokumente sowie sämtliche Unterlagen und Informationen zum Programm sind auf der Programmwebseite verfügbar unter http://www.swissuniversities.ch/isci. Dokumente zur Herleitung des Programms befinden sich im Archiv, ganz unten auf der Seite. 


\section{Ausgangslage, Vision und Mission $^{14}$}

Die rasch voranschreitende Entwicklung der Informationsund Kommunikationstechnologien bedingt die laufende Anpassung der informationstechnischen Grundlagen für Lehre und Forschung. Im Rahmen der internationalen $\mathrm{Zu}$ sammenarbeit unter Forschenden und Institutionen gewinnt dabei der Datenaustausch an Bedeutung. Auf die Herausforderungen im Bereich der Bewirtschaftung wissenschaftlicher Information reagieren die Schweizer Hochschulen bisher größtenteils eigenständig. Der große Veränderungsdruck legt jedoch ein vermehrt gemeinsames und arbeitsteiliges Handeln nahe.

Bis zum Jahr 2020 soll den Forschenden, Lehrenden und Lernenden in der Schweiz ein umfangreiches Grundangebot an digitalen Inhalten von wissenschaftlicher Relevanz zur Verfügung stehen. Ein Netzwerk von Services soll den einfachen Zugang zu Publikationen und Daten ermöglichen und Werkzeuge für deren Bearbeitung und Speicherung anbieten. Verfügbarkeit auf nationaler Ebene, Stabilität, Agilität und Wettbewerbsfähigkeit sollen diese Services auszeichnen.

Das Programm Wissenschaftliche Information fördert den Aufbau dieses nationalen Servicenetzwerks. Dank einer Bündelung der Kräfte sollen Synergien und Effizienzgewinne entstehen, die das starke Wachstum der Kosten im Bereich der wissenschaftlichen Information begrenzen und die Wettbewerbsfähigkeit der Schweiz als Spitzenstandort für Bildung und Forschung stärken. Eine anerkannte, handlungsfähige Organisation soll in Zukunft den dauerhaften Betrieb gewährleisten. ${ }^{15}$

Das Programm initiiert und steuert den Aufbau dieses Angebots durch gezielte Förderung. Ausgangspunkt sind 39 Umsetzungsmaßnahmen in den vier strategischen Hauptstoßrichtungen Publikationen, e-Science, Basis und Dienste, die im White Paper definiert wurden. ${ }^{16}$ Abb. 1 vermittelt eine Übersicht über die Inhalte der Hauptstoßrichtungen. Von 2013 bis 2016 standen für die Projekt-

14 SUK P-2: Bündelung der Kräfte in der wissenschaftlichen Information: nationale Strategie. 2014, Kapitel 1.2, 2.1 und 2.2 unter https:// www.swissuniversities.ch/fileadmin/swissuniversities/Dokumente/ Organisation/SUK-P/SUK_P-2/SUK_P-2_NationaleStrategie_201404 03_DE.pdf.

15 SUK P-2: Bündelung der Kräfte in der wissenschaftlichen Information, Kapitel 1.5.

16 SUK P-2: White Paper for a Swiss Information Provisioning and Processing Infrastructure 2020. 2015, Kapitel 5.3 unter https://www.s wissuniversities.ch/fileadmin/swissuniversities/Dokumente/Organis ation/SUK-P/SUK_P-2/WhitePaper_V1.1-DE.pdf. förderung Mittel in Höhe von 37 Mio. Franken zur Verfügung. ${ }^{17}$

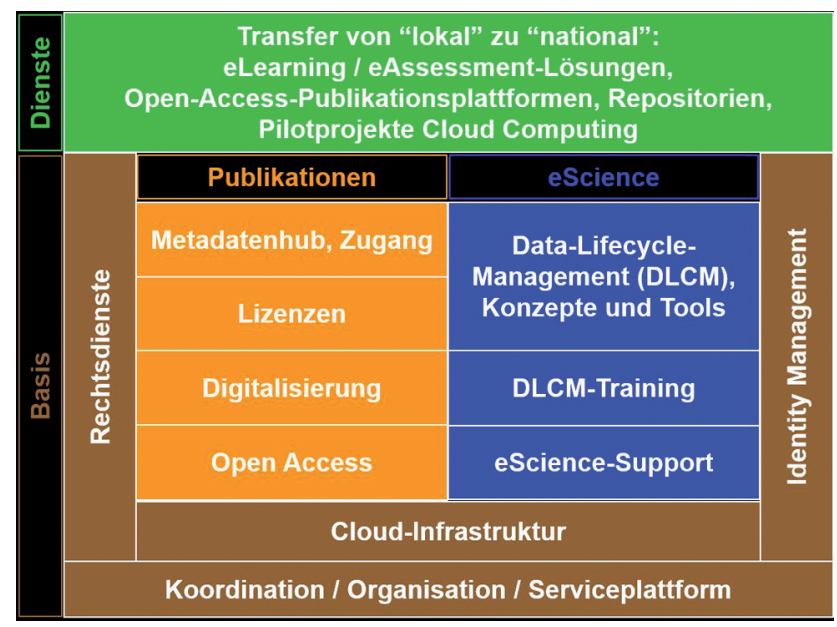

Abb. 1: Hauptstoßrichtungen und Inhalte des Programms Wissenschaftliche Information

\section{Projekteingaben und Mandate}

Das Ziel des Programms - gemeinsame nationale Services, die dezentral von Hochschulen und hochschulnahen Institutionen angeboten werden - bedingt, dass diese bereit sind, sowohl als Anbieter als auch als Bezieher an einem neuen Markt teilzunehmen. Diese Haltung soll schrittweise herbeigeführt werden: sowohl „top-down“, durch den Einbezug der Führungsgremien (Hochschulleitungen, swissuniversities) und der Förderorganisationen (SBFI, SNF), als auch „bottom-up“, durch die praktische Bereitstellung hochwertiger Angebote.

Weil dieser Prozess in einem föderalistischen System nur bedingt gesteuert werden kann, hängt der Programmerfolg wesentlich davon ab, ob die vorgeschlagenen Umsetzungsmaßnahmen sich mit eigenen Vorhaben der Hochschulen decken. Grundsätzlich werden die berechtigten Institutionen zur Eingabe von Projektanträgen aufgerufen. Vom Antragsteller werden Eigenleistungen in Höhe der beantragten Fördermittel erwartet (matching funds). Diese können tiefer sein, wenn der gesamtstrategische Nutzen des Projektes den Nutzen für die projektführende Institution überwiegt.

Einige Maßnahmen werden mit Partnern, die heute schon Leistungen für alle Hochschulen erbringen, direkt umgesetzt, namentlich mit SWITCH und dem Konsortium

17 Das Programm verfügte 2013-2016 insgesamt über Mittel in Höhe von CHF 45 Mio. CHF 8 Mio. wurden für die Weiterführung von Vorgängerinitiativen bis zur Definition der Strategie sowie für die Programmorganisation verwendet. 


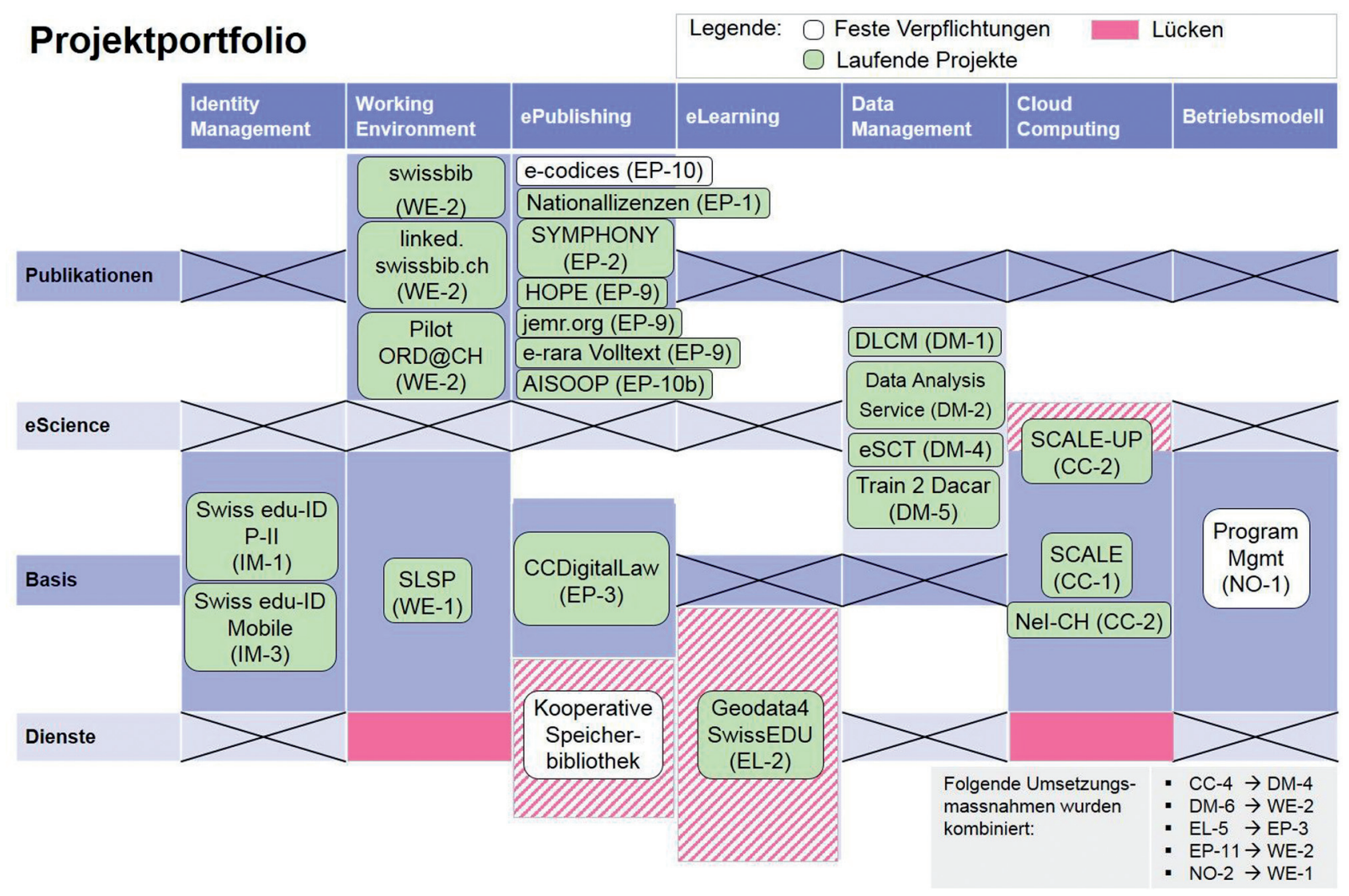

Abb. 2: Projektmatrix, Stand 31. Dezember 2015

der Schweizer Hochschulbibliotheken. Der Lenkungsausschuss hat zudem die Möglichkeit, Umsetzungsmaßnahmen durch Mandate gezielt anzustoßen. So beteiligte sich das Programm am Mandat des Schweizerischen Nationalfonds (SNF) zur Durchführung einer Finanzflussanalyse des wissenschaftlichen Publikationswesens in der Schweiz. Die Ergebnisse bilden eine Grundlage für die Entwicklung von Open Access an den Schweizer Hochschulen.

\section{Strategische Hauptstoßrichtungen und Projekte}

Im Strategieprozess des Programms wurde die Matrix (vgl. Abb. 2) aufgebaut, die es erlaubt, den Programmfortschritt $\mathrm{zu}$ verfolgen. Sie zeigt, in welchen Hauptstoßrichtungen (Y-Achse) Umsetzungsmaßnahmen für die im Programmantrag für SUK $\mathrm{P}-2^{18}$ beschriebenen Handlungsfelder (X-Achse) ausgeschrieben sind.

18 Projektgebundene Beiträge 2013-2016: Antrag auf ein SUK-Programm. 2012. Das Dokument ist im Archiv der Programmwebseite ver-
Die bewilligten Projekte sind im entsprechenden Feld der Matrix eingetragen, mit Angabe der Umsetzungsmaßnahme, die sie adressieren. Die roten und rot schraffierten Felder visualisieren die strategischen Bereiche, in denen noch keine bzw. nur wenige Dienste gefördert werden. ${ }^{19}$

\section{1 „Publikationen“: Lizenzierung, Open Access, Digitalisierung, Metadata/Search}

Die Hauptstoßrichtung Publikationen adressiert schwerpunktmäßig die Hochschulbibliotheken. Diese sind eingeladen, ein breit verfügbares elektronisches Grundangebot an wissenschaftlichen Publikationen aufzubauen. Die Aktivitäten dieser Hauptstoßrichtung sind maßgeblich getrieben vom Transfer analoger Publikationen in digitale

fügbar unter https://www.swissuniversities.ch/fileadmin/swissuni versities/Dokumente/Organisation/SUK-P/SUK_P-2/Demande_Progr amme_CUS_P-2_Information_Scientifique_DE.pdf.

19 Laufende und abgeschlossene Projekte sind auf den Unterseiten der Programmwebseite beschrieben unter http://www.swissuniversi ties.ch/isci. 
Arbeitsumgebungen und von der Transformation des wissenschaftlichen Publikationsmarktes zu Open Access. Ein wichtiges Anliegen ist die engere Vernetzung der Bibliotheksdienste mit den Anforderungen der Forschung. Für die Förderung wurden für 2013-2016 22 Mio. Franken budgetiert.

\subsubsection{Kollektive Lizenzierung}

Im Projekt Nationallizenzen des Konsortiums der Schweizer Hochschulbibliotheken wurden im Frühling 2016 mit De Gruyter Journals, Cambridge sowie Oxford University Press Journals erste Backfile-Archive für den nationalen Zugang lizenziert. Durch die Anbindung an die Konsortiallizenzen für Current Content sollen lückenlose „Rolling Archives“ erzielt werden, zudem sollen Mehrwerte auf Current Content wie Open-Access-Klauseln oder Hybridkompensation (Offsetting) verhandelt werden. Langzeitarchivierung, Metadatenintegration und eine moderne $\mathrm{Au}-$ thentifizierungslösung in Zusammenarbeit mit SWITCH sind Bestandteile des Projektes. Es ist in den Bibliotheken breit abgestützt und bindet ausländische Expertise ein..$^{20}$

Ab 2017 will das Konsortium die Projekterfahrungen für innovative Lizenzen nutzen. Vor allem aber soll eine dauerhafte Geschäftsform gegründet werden: Das Konsortium, das 2015 Lizenzen in Höhe von 27,8 Mio. Franken verhandelte, ist bis jetzt eine Projektorganisation.

\subsubsection{Open Access}

Das Programm Wissenschaftliche Information bietet den Hochschulen mehrere Umsetzungsmaßnahmen zur Förderung von Open Access an, die bisher nur geringes Echo fanden. ${ }^{21}$ Das Programm unterstützt gegenwärtig den Umzug des Journals of Eye Movement Research (JEMR) auf die OJS-Plattform der Universität Bern ${ }^{22}$ und den Aufbau einer OJS-Plattform an der Universität Zürich (Projekt: Hope for Open Access). Bislang fehlen in der Schweiz Trägerschaften für gemeinsame Infrastrukturen. Gleichzeitig wird die Beteiligung der Schweiz an den international vermehrt koordinierten Initiativen für Open Access dringender. So steht Open Access im Zuge der Anstren-

20 Vgl. http://www.consortium.ch/national-lizenzen.

21 Präsentation der Programmleitung an den Open-Access-Tagen in Zürich, vom 07.09.2015, unter https://open-access.net/fileadmin/o at/oat15/slides/06-Schneider-Gabi-neu-OpenAccessTage_SUK_P-2_S ept7.pdf.

22 Vgl. https://www.bop.unibe.ch/jemr. gungen in Richtung Open Science auf der Agenda der Europäischen Union. ${ }^{23}$

Vor diesem Hintergrund ersuchte der Staatssekretär des SBFI Ende 2015 swissuniversities, sich unter Einbezug des SNF, „an der Erarbeitung einer gesamtschweizerischen Strategie für Open Access federführend zu beteiligen“. Er formulierte folgenden Handlungsbedarf:

- Gezielte Förderung von Open Access als übergeordnetes Ziel,

- Schaffung von Kostentransparenz bei den öffentlichen Ausgaben im Bereich wissenschaftliches Publizieren (Finanzflussanalyse),

- Koordination der Aktivitäten der Stakeholder, seitens der Hochschulen namentlich auch unter Einbezug der Hochschulbibliotheken. ${ }^{24}$

Swissuniversities hat Anfang 2016 mit dem Strategieprozess begonnen. ${ }^{25}$ Es besteht Hoffnung, dass eine gemeinsame Strategie der Hochschulen eine bessere Grundlage für konkrete Umsetzungsprojekte in der Förderperiode von 2017 bis 2020 bietet.

Für die geforderte Kostentransparenz hat der SNF gemeinsam mit SUK P-2 eine Finanzflussanalyse des wissenschaftlichen Publikationswesens in der Schweiz mandatiert, die Szenarien für eine Umstellung auf Open Access aufzeigen soll. Die Analyse soll bis Ende 2016 vorliegen.

Ein Schlüssel zur Bewältigung der Umwälzungen im wissenschaftlichen Publikationswesen ist das Konsortium: Unterstützen die primären Konsortialpartner eine stärkere Organisation mit gemeinsamer Verhandlungsführung gegenüber den großen Wissenschaftsverlagen, kann die Transformation des Marktes in Richtung Open Access auch von der Schweiz mit angeschoben werden.

\subsubsection{Digitalisierung}

Im März 2015 verabschiedete das Programm ein dediziertes Strategiedokument zum Umsetzungsbereich Digitalisierung. ${ }^{26}$ Es konkretisiert den Wunsch nach einer breiteren

23 Digital Agenda for Europe: Open Science unter http://ec.europa. eu/digital-agenda/en/open-science.

24 Brief des Staatssekretärs für Bildung, Forschung und Innovation vom 04.12.2015 unter https://www.swissuniversities.ch/fileadmin/ swissuniversities/Dokumente/Hochschulpolitik/151204_Brief_SBFI OA nationale_Strategie_Swissuniversities_sign.pdf. 25 Über den Stand der Arbeiten informiert die Webseite unter https:// www.swissuniversities.ch/de/themen/hochschulpolitik/open-access. 26 SUK P-2: Hauptstoßrichtung Publikationen, Umsetzungsmaßnahme EP-10, Digitalisierung: angepasste Strategie und Umsetzungsmaß- 
Partizipation von Gedächtnisinstitutionen und Forschung an den Plattformen, die z.B. im Rahmen von E-lib.ch aufgebaut wurden. Die Implementierung von Standards (technische Standards und Metadaten), die Verbesserung der Interoperabilität und die Entwicklung von Tools für die Forschung sollen gefördert werden. Ein Kompetenzzentrum könnte in Zukunft neben Digitalisierungsfachleuten auch Forschende beraten, und ein Fonds zur Finanzierung von Retrodigitalisierungsvorhaben könnte es Forschungsförderung und Dienstleistern ermöglichen, gemeinsam über Retrodigitalisierungsvorhaben von nationaler Relevanz zu entscheiden.

Die Erarbeitung des Strategiedokumentes erfolgte zeitgleich mit einem Call für Editionsprojekte des SNF sowie einem Pilotprojekt der Schweizerischen Akademie der Geistes- und Sozialwissenschaften (SAGW) und der Universität Basel zur Einrichtung eines Daten- und Dienstleistungszentrums für die Geisteswissenschaften (Data and Service Center for the Humanities, DaSCH). ${ }^{27}$ Der Austausch mit diesen Vorhaben machte Anforderungen an die Digitalisierung von Seiten der Digital Humanities deutlich. Mit dem Projekt NIE-INE (nationale Infrastruktur für Editionen), das Mitte 2016 bewilligt wurde, fördert das Programm deshalb ein Kooperationsvorhaben der Universitäten Basel, Zürich, Bern und Genf sowie der SAGW zum Aufbau einer technischen Architektur für die Bearbeitung digitaler kritischer Editionen. ${ }^{28}$ Im Projekt histHub erarbeiten vier Unternehmen der SAGW einen Hub für historische Entitäten als Linked Open Data. Eine Schweizer Besonderheit wird hier die Mehrsprachigkeit der Vokabulare sein.

Das Projekt AISOOP analysiert, wie die bestehenden Bibliotheksplattformen im Sinne der Strategie weiter zu entwickeln wären. Die Virtuelle Handschriftenbibliothek Schweiz (e-codices), die Plattform für digitalisierte handschriftliche Quellen aus Schweizer Bibliotheken und Archiven (e-manuscripta) sowie die Plattform für digitalisierte Drucke aus Schweizer Bibliotheken (e-rara) werden vom Programm weiterhin gefördert. ${ }^{29}$

nahmen, vom 31. März 2015 unter https://www.swissuniversities.ch/ fileadmin/swissuniversities/Dokumente/Organisation/SUK-P/SUK_ P-2/SUK_P-2_StrategieDigitalisierung_DE.pdf.

27 Zum Aufbau von DaSCH vgl. http://www.sagw.ch/sagw/laufen de-projekte/DaSCH.html.

28 Die Projektleitung hat das Forum Edition und Erschließung der Universität Basel. Informationen hierzu unter http://www.fee.uni bas.ch.

29 Vgl. www.e-codices.ch, www.e-manuscripta.ch und www.e-rara. ch. Für das Abstract von AISOOP vgl. https://www.swissuniversities. $\mathrm{ch} /$ fileadmin/swissuniversities/Dokumente/Organisation/SUK-P/SU K_P-2/Abstract_AISOOP.pdf.

\subsubsection{Suchlösungen/Metadaten-Hubs}

Mit den Projekten swissbib und linked.swissbib ${ }^{30}$ fördert SUK P-2 die Weiterentwicklung der Metadatenkomponenten des Schweizer Metakatalogs swissbib.ch. Das Programm investierte zudem in ein Pilotprojekt für einen Metadatenhub für Forschungsdaten (Pilot ORD@CH). ${ }^{31}$ Während die Zukunft von swissbib in Richtung Konsolidierung mit dem Projekt Swiss Library Service Platform (SLSP) in der Hauptstoßrichtung Basis deutet, wird openresearchdata seine Wettbewerbsfähigkeit im Vergleich mit internationalen Vorhaben unter Beweis stellen müssen.

\section{2 „E-Science“: Data Lifecycle Management, Training und Support}

Die Hauptstoßrichtung e-Science unterstützt die Bewirtschaftung von Forschungsdaten. Sie erarbeitet standardisierte Prozesse für den Zugang, die Bearbeitung, die Nachnutzung und die Archivierung. Supportdienste unterstützen Forschende dabei, Methoden und Knowhow den verfügbaren Rechenleistungen entsprechend zu skalieren. Das Budget in Höhe von 3 Mio. Franken spiegelt die Erwartung, dass kooperative Dienste in e-Science erst am Anfang ihres Lebenszyklus stehen.

\subsubsection{Data Lifecycle Management (DLCM)}

Das Großprojekt Data Lifecycle Management (DLCM) ${ }^{32}$ erarbeitet Konzepte und exemplarische Lösungen für den Lebenszyklus von Forschungsdaten. Die Partner, die Eidgenössischen Technischen Hochschulen, die Universitäten Genf, Lausanne, Basel und Zürich, die Fachhochschule Genf und SWITCH, entwickeln die Inhalte in einem Bogen über West- und Deutschschweiz, Geistes- und Naturwissenschaften sowie IT-Services, Scientific IT und Bibliotheken.

Die nachstehende Grafik (Abb. 3) zeigt die Arbeitspakete und Inhalte des Projektes.

Von 2017 bis 2020 soll das Programm lokal etablierte Dienste dabei unterstützen, auf der Basis der erarbeiteten Konzepte schweizweit verfügbare Angebote zu entwickeln. Data-Management-Dienste, lokale Werkzeuge

30 Abstract unter http://campus.hesge.ch/id_bilingue/projekte/lin kedswissbibch/default.asp.

31 Das Projekt ging Mitte 2015 mit einer Beta-Plattform online. Siehe http://openresearchdata.ch.

32 Projektseite unter http://www.dlcm.ch. 
und Knowhow im Bereich e-Science sollen einer größeren Forschungsgemeinde angeboten werden. Pilotprojekte, die diese Angebote übernehmen, sollen gefördert werden.

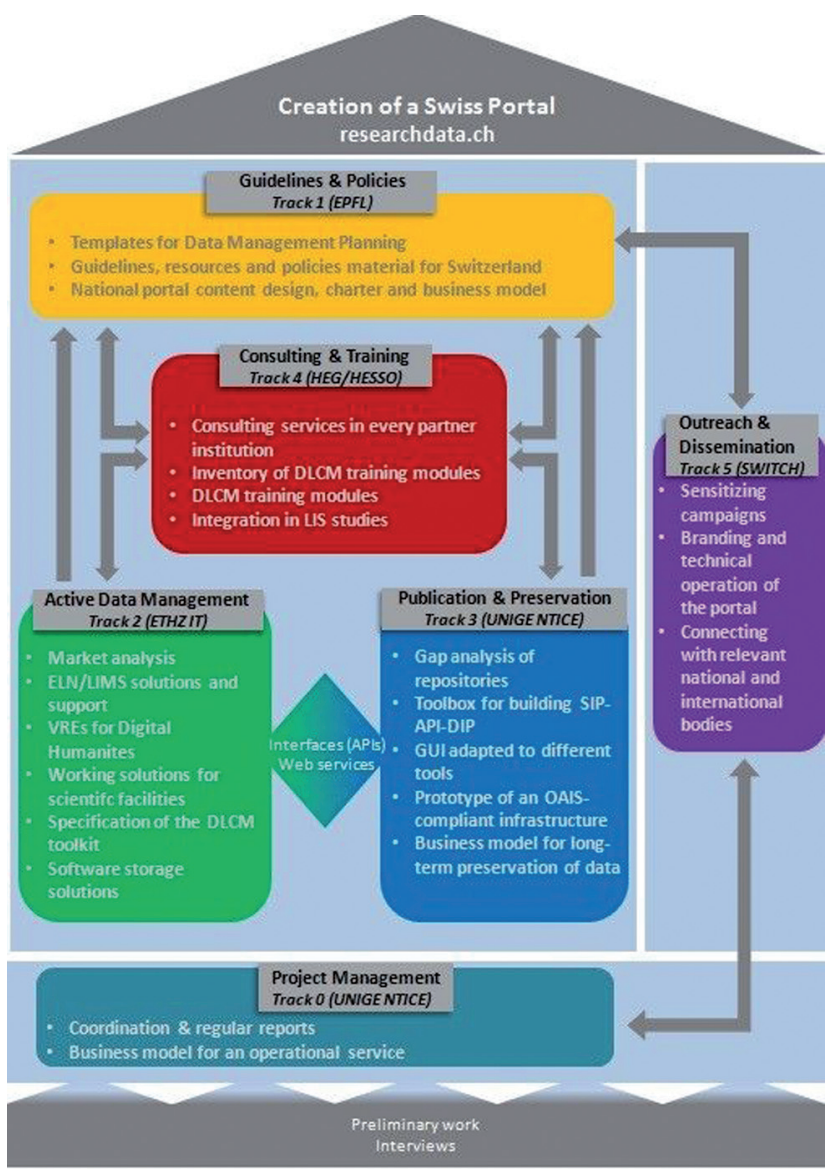

Abb. 3: Projekt Data Lifecycle Management (DLCM), Arbeitspakete und Inhalte

\subsubsection{Training}

Ende 2016 wird das Projekt Train 2 Dacar seine Ausbildungsmodule getestet und konsolidiert haben. ${ }^{33}$ Dieses Ausbildungsangebot entsteht an den Fachhochschulen und ermöglicht Forschenden und Mitarbeitenden von Bibliotheken und IT-Services eine Weiterbildung in Richtung Data-Management-Support. Das Programm will den Aufbau ähnlicher Angebote weiterhin unterstützen.

33 Abstract unter http://campus.hesge.ch/id_bilingue/projekte/trai n2dacar/default.asp.

\subsubsection{E-Science-Support}

„Shorter time to solution“ ist der Inhalt des Projekts eScience Coordination Team (eSCT), das einen Supportdienst für Forschende aufbaut. ${ }^{34}$ Das Projekt macht das Expertenwissen und die Werkzeuge der führenden Universitäten, z. B. zur Bearbeitung großer Datenmengen, auch kleineren Hochschulen zugänglich.

Der Data Analysis Service, den das Paul Scherrer Institut (PSI) für seine Kunden aufbaut, hat zum Ziel, die Analyse, Bearbeitung und Speicherung der großen Datenmengen, welche die neuen Forschungsinfrastrukturen generieren, online zu ermöglichen. Die übertragbaren Konzepte und Methoden sollen der Forschungsgemeinde zur Verfügung gestellt werden.

\section{3 „Basis“: Kernstücke des künftigen Service-Networks}

Der Aufbau und die Anpassung technischer und organisatorischer Grundlagen für die Zusammenarbeit von Bibliotheken, IT-Services und Scientific IT sind Voraussetzung für die Verankerung des Programms. Entsprechende Projekte werden in der Hauptstoßrichtung Basis gefördert. $\mathrm{Zu}$ Basis gehören der Aufbau einer landesweit flexibel nutzbaren Cloud-Infrastruktur, die Weiterentwicklung der Identity-Management-Lösung von SWITCH, Kompetenzzentren im Bereich Urheber- und Datenrecht sowie zur Unterstützung von Open Access und Open Data, und eine nationale Organisation, die langfristig als Drehscheibe für die aufgebauten Dienste und Infrastrukturen agiert. Das geschätzte Fördervolumen für 2013 bis 2016 beträgt 7 Mio. Franken.

\subsubsection{Identity Management: SWITCHaai „new generation“}

SWITCH bietet heute mit SWITCHaai eine föderierte Identity-Management-Lösung an, die mit den Zugangsdaten der eigenen Hochschule den hochschulübergreifenden Zugriff auf Webressourcen erlaubt. Das Projekt Swiss edu-ID entwickelt diese Lösung zu einem nutzerzentrierten IdentityManagement weiter, das eine lebenslange akademische Identität anbietet. ${ }^{35}$ Im Verlauf des Jahres 2016 ist die Swiss edu-ID bereit für die Anbindung an die lokalen Kontover-

34 Projektseite unter http://www.science-it.ch. 35 Swiss edu-ID: the academic identity made in Switzerland unter https://projects.switch.ch/eduid. 
waltungen der Hochschulen und ihrer Ressourcen. Beispielsweise wird die Autorisierung für die Schweizer Nationallizenzen auf der Swiss edu-ID basieren.

\subsubsection{Swiss Library Service Platform (SLSP)}

Noch bis Februar 2017 läuft die Konzeptionsphase für die Swiss Library Service Platform (SLSP). Der Austritt des Kantons Waadt aus dem Westschweizer Bibliotheksverbund RERO und der Umstand, dass die integrierten Bibliothekssysteme der großen Hochschulbibliotheken das Ende ihres Lebenszyklus erreichen, hat zu einer breit abgestützten Initiative geführt, die Schweizer Bibliotheksverbünde technisch und organisatorisch auf eine neue Grundlage $\mathrm{zu}$ stellen. Während die Kosten für die lokale Betriebserneuerung in die Zuständigkeit der Träger fällt, wird das Programm Projekte fördern, die zu einer gemeinsamen Lösung und einer neuen Grundlage für die Dienstleistungen der Hochschulbibliotheken führen.

\subsubsection{Urheber- und Datenrecht}

Die Digitalisierung verändert die rechtlichen Grundlagen für die Arbeit in Forschung und Lehre. Im Dezember 2015 wurde der Aufbau eines Kompetenzzentrums für digitales Recht (CC Digital Law) unter Federführung der Università della Svizzera italiana (USI) bewilligt. In einem Vorprojekt wurde dieses u. a. durch die Konsultation der Rechtsdienste der Hochschulen abgesichert. Die erste Projektphase (bis Juni 2017) fokussiert auf Ausbildungsmodule in den Bereichen Urheberrecht (Rechte, Lizenzen, Open Access) und die Findung eines nachhaltigen Geschäftsmodells. In einer nächsten Phase soll das Projekt um den Bereich Datenrecht erweitert werden.

\subsubsection{Cloud-Infrastruktur}

Das bereits abgeschlossene Projekt SCALE baute eine national verfügbare Cloud-Infrastruktur (IaaS) auf, die inzwischen unter der Bezeichnung „SWITCHengines“ im Einsatz ist. ${ }^{36}$ In einem nächsten Schritt entwickelt das Projekt SCALE-UP eine Reihe einfach zu nutzender Dienste (PaaS) für Forschung und Lehre. ${ }^{37}$ Nel-CH ermöglicht Schweizer Forschenden als nationale Zertifizierungsstelle und Link

36 SWITCHengines: http://www.switch.ch/de/engines. 37 Projektseite unter https://projects.switch.ch/scale-up. zur europäischen Grid- und Cloud-Infrastruktur den $\mathrm{Zu}$ gang zu den Rechenkapazitäten der E-Infrastruktur-Initiative EGI der Europäischen Union. ${ }^{38}$

\section{4 „Dienste“: konsolidieren und erweitern}

Die Hauptstoßrichtung Dienste übernimmt die Investitionskosten für die Öffnung von Services sowie die Einstiegskosten neuer Hochschulen zu solchen Lösungen. Sie unterstützt den Ausbau lokaler Infrastrukturen wie Repositorien, Publikationsplattformen oder E-Learning-Werkzeuge zu nationalen Services. Dienste fördert zudem kooperative Lösungen auf der Basis von Services, die in anderen Hauptstoßrichtungen aufgebaut werden (z. B. Pilotprojekte zur Nutzung der Cloud-Infrastruktur), und zur Integration von Services in Arbeitsumgebungen. Hier ist auch Raum für kooperative Pilotprojekte in neuen Anforderungsbereichen. Das Fördervolumen wurde auf 5 Mio. Franken veranschlagt.

\subsubsection{Erweiterung als Querschnittsprinzip}

Mit dem beschriebenen Ansatz wollte das Programm in erster Linie eine größere Partizipation an E-Learning-Werkzeugen bewirken. In der Fortsetzungsphase könnte die Förderung gemeinsamer Lösungen der Hochschulen als Querschnittsprinzip in allen Strategiebereichen angestrebt werden, um eine größere Breitenwirkung zu erzielen.

Ein Beispiel ist das Projekt Geodata4SwissEDU. Sein Ziel ist ein leistungsfähiger Service für das Auffinden, den Zugriff, die Präsentation, den Download und die Verarbeitung von Geoinformationen für Ausbildung und Forschung an Schweizer Hochschulen. Geodata4SwissEDU führt lokale Vorhaben der ETH Zürich und der Hochschule für Technik Rapperswil (HSR) zusammen.

\subsubsection{Kooperative Speicherbibliothek Schweiz}

Der Hauptstoßrichtung Dienste wurde der Aufbau der „Kooperativen Speicherbibliothek Schweiz“ zugeteilt, deren Förderung 2013 weitergeführt wurde. Die Speicherbibliothek im luzernischen Büron nahm am 1. Februar 2016 den Betrieb auf und wurde am 24./25. Juni offiziell eröffnet. ${ }^{39}$

38 Vgl. http://www.egi.eu. 39 https://de.wikipedia.org/wiki/Kooperative_Speicherbibliothek_ Schweiz. 


\section{Ausbau der Projektcluster (2017 bis 2020)}

Dem Programm Wissenschaftliche Information ist es bereits gelungen, die Dienstleister der Hochschulen (Bibliotheken, IT-Services und Scientific IT) zu vernetzen und in starken Kollaborationen zusammenzubringen. Die Hälfte aller universitären Hochschulen und zwei von sieben Fachhochschulen leiten eines oder mehrere Projekte. Alle kantonalen Universitäten, beide ETHs sowie sechs von sieben Fachhochschulen sind an Projekten beteiligt.

Die Erarbeitung einer nationalen Strategie, ihre konsequente Verfolgung bei der Projektauswahl und die Verankerung des Programms haben rund zwei Jahre in Anspruch genommen. Bis Ende 2016 wurden 23 Projekte gefördert. Konnten bei der Ersteingabe im April 2014 erst fünf von 42 eingereichten Anträgen bewilligt werden, bewegt sich die Erfolgsquote nach vier Evaluationen bei ca. $30 \%$. Um den Aufbau nicht zu bremsen, wurden in Absprache mit dem SBFI ab 2015 Projekte mit Laufzeiten über die aktuelle Finanzierungsperiode hinaus bewilligt. Seit Sommer 2015 ist die Grundlage für eine schrittweise Konsolidierung der aufgebauten Service-Cluster gegeben.

Von 2017 bis 2020 sollen die Service-Cluster als Umsetzungsschwerpunkte weiterentwickelt und konsolidiert werden. Große Erwartungen richten sich an das Projekt Swiss Library Service Platform (SLSP). Neben der Aussicht, dass eine neue Plattform, geführt von einer breiter abgestützten Service-Organisation, bibliothekarische Routinearbeiten effizienter erledigen kann als die kleinräumigen Bibliotheksverbünde, besteht die Aussicht, Dienste wie swissbib, das Konsortium der Schweizer Hochschulbibliotheken oder die Digitalisierungsplattformen mittelfristig zu integrieren. ${ }^{40}$

Exemplarisch für die erfolgreiche Vernetzung und die gegenseitige Abstimmung ist folgendes Beispiel: Das Projekt Data Lifecycle Management (DLCM) erarbeitet Best Practices und Tools für die Verwaltung und den Erhalt von Forschungsdaten. Das eScience Coordination Team (eSCT) übernimmt diese Kenntnisse und vermittelt die Unterstützung in IT-Fragen für Forschende über Organisationsgrenzen hinweg. Dabei werden lokale Dienste der Hochschulen genutzt, aber auch Cloud-Services, die durch die Projekte SCALE und SCALE-UP von SWITCH oder vom Projekt NeI$\mathrm{CH}$ angeboten werden. Train2Dacar erarbeitet Trainingsmodule und Kurse für das Forschungsdaten-Management,

40 Der Fortschritt von SLSP kann im Projektblog unter http://www. slsp.ch verfolgt werden. während Pilot-ORD@CH mit openresearchdata.ch eine Beta-Plattform für Metadaten aufgebaut hat, die das Angebot für das Forschungsdaten-Management abrunden könnte.

\section{Von der Programmorganisation zu einer „nationalen Organisation“}

Die nationale Strategie für SUK P-2 bezeichnet die Programmorganisation als Ausgangspunkt für den Aufbau einer dauerhaften Koordinationsstelle und einer ServicePlattform für die aufgebauten Dienste. ${ }^{41}$ Die geplante nationale Organisation soll den Servicekatalog führen, die Einhaltung von Vereinbarungen überprüfen, Richtlinien und Schnittstellen definieren und den Einsatz von Finanzmitteln koordinieren. ${ }^{42}$ Da die Voraussetzungen der drei Hochschultypen in Bezug auf die Beteiligung an Diensten sehr unterschiedlich sind, zeichnet sich die Findung eines anwendbaren Geschäftsmodells und einer Rechtsform als größte Herausforderung ab.

Die konkreten Arbeiten zum Aufbau einer nationalen Governance, die eine dynamische, hochschulübergreifende Planung und Finanzierung von Diensten unterstützen soll, haben in der zweiten Jahreshälfte 2016 begonnen. Sie werden nur mit Unterstützung der Steuergremien (Lenkungsausschuss, swissuniversities, Schweizerische Hochschulkonferenz, SBFI), der Partner und der Stakeholder Erfolg haben. Gleiches gilt für die Herausbildung von Vereinbarungen (Policies), die von den Hochschulen gemeinsam getragen werden. Das Beispiel Open Access zeigt, dass Umsetzungsmaßnahmen wie SUK P-2 sie vorsieht erst greifen, wenn Hochschulen ihre Initiativen zur Entwicklung des kostenintensiven Bereichs der wissenschaftlichen Information koordinieren. Die geplante Organisation könnte kooperative Transformationsprozesse unterstützen.

\section{Programmfortsetzung 2017 bis 2020}

Am 27. Mai 2016 entschied die Schweizerische Hochschulkonferenz, das Programm Wissenschaftliche Information in der Finanzierungsperiode 2017 bis 2020 fortzusetzen. In der neuen Förderperiode sind Investitionen in Höhe von

41 SUK P-2: Nationale Strategie, Kapitel 1.5 und 4. Die Grundlagen des Betriebsmodells wurden im White Paper, Kapitel 4.7, erarbeitet. 42 SUK P-2: Nationale Strategie, Kapitel 4. 
30 Mio. Franken geplant, zusätzlich wurden die bereits bewilligten Projekte, die über 2016 hinaus laufen, mit Beiträgen in Höhe von 7 Mio. Franken abgesichert.

In einer Ex-ante-Evaluation der Vorhaben für projektgebundene Beiträge erhielt die Fortsetzung von SUK P-2 die höchste Anzahl Punkte bezüglich Systemrelevanz. Diese wurde mit Vorteilen im internationalen Wettbewerb, der Generierung zusätzlicher Steuerungsinformationen und einem größeren gesellschaftlichen Bedürfnis deutlich bejaht. Das Vorhaben, das einem ausgewiesenen Bedürfnis der Hochschulen entspreche, wurde als Aufgabe von gesamtschweizerischer hochschulpolitischer Bedeutung nach Art. 59 HFKG gewertet. ${ }^{43}$ Es soll die Bildung von Kompetenzzentren fördern und mittelfristig eine Portfoliobereinigung ermöglichen.

Mit der Entwicklung von SUK P-2 zeichnen sich Überschneidungen mit anderen Forschungsinfrastrukturen ab. Neben bestehenden Infrastrukturen und Dienstleistern wie SWITCH, FORS (Swiss Centre of Expertise in the Social Sciences), CSCS (Centro Svizzero di Calcolo Scientifico) oder Vital-IT (Infrastructures for Life-Sciences) verzeichnet die Schweizer Roadmap für Forschungsinfrastrukturen 2015 mehrere neue FIS, die mit den strategischen Feldern des Programms überlappen. Beispiele sind die „Initiative for Data Science in Switzerland“ (IDSS) im ETH-Bereich, das „Swiss Digital Humanities Center“ (SDHC) der Universität Basel, die „Swiss Art Research

43 Schenker-Wicki, Andrea: Projektgebundene Beiträge nach HFKG, Ex-ante-Evaluation der von den Schweizerischen Hochschulen eingegebenen Projekte für die Beitragsperiode 2017-2020, 2015, S. 13-14 [internes Dokument].
Infrastructure“ (SARI) der Universität Zürich oder BioMedIT „Information and computational service infrastructure network to support biomedical research in Switzerland“ des Swiss Institute of Bioinformatics. ${ }^{44}$ Anspruchsvoll ist dabei vor allem die Abgrenzung generischer, allgemein gültiger Grundlagen für die Verarbeitung wissenschaftlicher Information von disziplinären Vorhaben. Die geplante nationale Organisation steht in einem komplementären Verhältnis zu Dienstleistungsanbietern wie SWITCH, der Schweizerischen Akademie der Geistes- und Sozialwissenschaften (SAGW) oder dem Schweizerischen Nationalfonds zur wissenschaftlichen Forschung. Ist die Portfoliobereinigung ernst gemeint, erfordert dies in Zukunft eine engere Zusammenarbeit auf der Ebene der Schweizer Hochschulplanung.

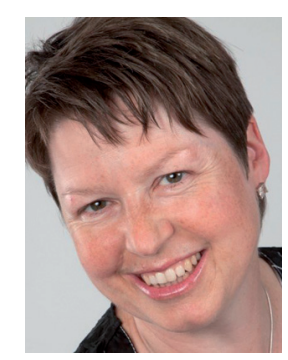

\section{Gabi Schneider}

Stellvertretende Programmleiterin Programm „Wissenschaftliche Information: Zugang, Verarbeitung und Speicherung“ Swissuniversities c/o Universitätsbibliothek Basel Schönbeinstr. 18-20

CH-4056 Basel

Schweiz

gabi.schneider@swissuniversities.ch

44 Schweizer Roadmap für Forschungsinfrastrukturen 2015 mit Blick auf die BFI-Botschaft 2017-2020, Anhang A. Vgl. Fußnote 4. 